\title{
Regulation of hematopoiesis in endosteal microenvironments
}

\author{
Noboru Asada $\cdot$ Yoshio Katayama
}

Received: 4 April 2014/ Accepted: 8 April 2014/Published online: 24 April 2014

(C) The Japanese Society of Hematology 2014

\begin{abstract}
After birth, the hematopoietic system develops along with bone formation in mammals. Osteolineage cells are derived from mesenchymal progenitor cells, and differentiate into several types of bone-forming cells. Of the various types of cell constituents in bone marrow, osteolineage cells have been shown to play important roles in hematopoiesis. Early studies have identified osteoblasts as a hematopoietic stem cell niche component. Since that time, the role of endosteal microenvironment as a critical regulator of hematopoietic stem/progenitor cell (HSC/ HPC) behavior has been appreciated particularly under stress conditions, such as cytokine-induced HSC/HPC mobilization, homing/engraftment after bone marrow transplantation, and disease models of leukemia/myelodysplasia. Recent studies revealed that the most differentiated osteolineage cells, i.e., osteocytes, play important roles in the regulation of hematopoiesis. In this review, we provide an overview of recent advances in knowledge of regulatory hematopoietic mechanisms in the endosteal area.
\end{abstract}

\section{N. Asada $(\square)$}

Hematology, Oncology and Respiratory Medicine, Okayama

University Graduate School of Medicine, Dentistry and

Pharmaceutical Sciences, 2-5-1 Shikata-cho, Kita-ku,

Okayama 700-8558, Japan

e-mail: Noboru.Asada@einstein.yu.edu

Present Address:

N. Asada

Albert Einstein College of Medicine, Jack and Pearl Resnick Campus, 1301 Morris Park Avenue, Bronx, NY 10461, USA

Y. Katayama

Hematology, Department of Medicine, Kobe University

Hospital, 7-5-2 Kusunoki-cho, Chuo-ku, Kobe 650-0017, Japan
Keywords Osteolineage cells · Osteoblasts · Osteocytes · Hematopoiesis · Niche

\section{Introduction}

The hematopoietic organ, bone marrow (BM), is encapsulated by highly mineralized bone. Given their anatomic proximity, bone cells are thought to have a functional relationship with hematopoietic cells. To date, numerous studies have shown a relationship between bone tissue and BM. Osteolineage cells are derived from mesenchymal progenitor cells (MPCs) that have the capacity to differentiate into cells of three different lineages-chondrocytes, adipocytes, and osteoblasts-in vivo. Various stromal cell types, including CXCL12 abundant reticular cells [1], Nestin-GFP positive cells [2], Prx-1-Cre-derived cells [3, 4], and NG2+ pericytes [5], are reported to exhibit MPC activity. These cells have also been shown to be essential constituents of the hematopoietic stem/progenitor cell (HSC/HPC) niche with some degree of redundancy. Osteolineage cells are divided into MPCs, pre-osteoblasts, osteoblasts, and osteocytes, as determined by their stage of differentiation in vivo (see Fig. 1). During the process of differentiation, unique expression patterns of transcription factors identify these cells at each differentiation stage. Runt-related transcription factor 2 (Runx2) and Osterix (OSX) are the master regulator genes that lead MPCs to differentiate into the osteolineage [6, 7] and the high expression of these two genes is a hallmark of pre-osteoblasts [7, 8]. Bone-lining osteoblasts are a more mature subset and play major roles in osteogenesis by producing collagen. These mature osteoblasts are characterized by the expression of osteocalcin, alkaline phosphatase, and osteopontin. Osteoblasts are known to adopt three fates: (1) 


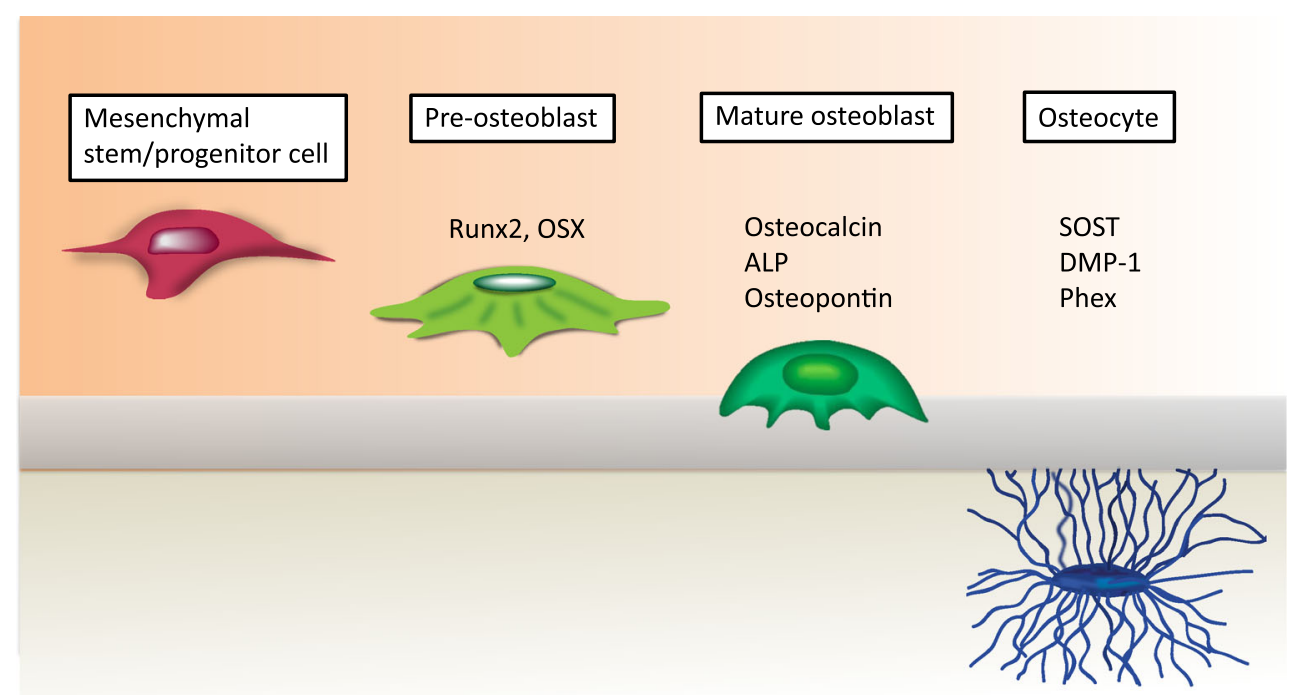

Fig. 1 Differentiation of osteolineage cell

become embedded within the matrix and convert into terminally differentiated osteocytes, (2) become inactive bone-lining cells [9], or (3) undergo apoptosis [10]. In this lineage, osteocytes are the most terminally differentiated cell type. A number of important roles for osteocytes in bone biology, such as mechanosensation and mineral metabolism, have been identified in previous studies [11]. A series of recent studies, including one by our group, has revealed the importance of osteocytes in the regulation of hematopoiesis [12-14]. In this review, we provide an overview of advances in our knowledge of regulatory mechanisms in the hematopoietic system by osteolineage cells in the endosteal area.

\section{Osteoblast progenitor (Pre-osteoblast) and osteoblast}

Early studies showed that bone-forming osteoblasts have the capacity to support immature hematopoietic cells in vitro [15]. The following studies used murine experimental models to show that intravenously transplanted HSCs homed to the BM and localized in the vicinity of endosteal area, suggesting that HSC niche-contributing cells are found near the border between BM and bone. In 2003, two groups using different mouse models demonstrated that osteolineage cell activation led to an increase in HSC number in vivo $[16,17]$. One group used transgenic mice, in which parathyroid hormone (PTH) signaling is constitutively active, or systemic PTH administration to activate osteolineage cells [16]. The other analyzed mutant mice with conditional inactivation of bone morphogenetic protein (BMP) receptor type IA [17]. In both models, the numbers of osteoblasts and HSCs were positively correlated. These findings shed light on the role of osteolineage cells as a HSC niche. It has also been reported that among the heterogeneous endosteal osteoblasts, spindleshaped N-cadherin positive osteoblastic (SNO) cells establish a niche for quiescent HSCs [17]. The results of an in vivo study, in which the number of osteoblastic cells, excluding SNO cells, were manipulated by strontium treatment and showed no remarkable change in HSC numbers, further supporting the importance of SNO cells for the HSC niche [18]. For endosteal osteoblastic cells, Nakamura et al. [19] subdivided endosteal cells by their expression of activated leukocyte cell-adhesion molecule (ALCAM) and Sca-1, and showed that ALCAM+ Sca-1osteoblastic cells harbor a greater capacity to support HSCs than other osteoblastic cell fraction in vitro.

Factors that mediate the relationship between osteoblast (so-called "osteoblastic niche") and quiescent HSCs have been reported by several studies. The interaction of angiopoietin-1, which is expressed by osteoblastic niche, with its receptor Tie 2 on HSCs has been shown to be critical for HSC quiescence [20]. Subsequent studies reported that thrombopoietin/MPL signaling is a niche factor that promotes HSC quiescence [21, 22]. More recently, researchers provided evidence that Flamingo and Frizzled 8-mediated noncanonical Wnt signaling in N-cadherin-positive osteoblastic niche cells promote quiescence of HSCs in homeostasis [23]. $\left[\mathrm{Ca}^{2+}\right]$ has also been shown to be an important factor to recruit HSCs to endosteum via calciumsensing receptors following intravenous transplantation, in which bone remodeling actively produces $\left[\mathrm{Ca}^{2+}\right]$ locally [24].

Osteoclasts are derived from HSCs in the BM, and are indispensable for bone modeling and remodeling. 
Osteoclasts interact with osteoblasts through various cell surface molecules and secreted proteins. Recent studies have implicated osteoclasts in HSC niche regulation. Activation of osteoclasts enhances mobilization of HSCs/ HPCs through the degradation of Cxcl12, stem cell factor (SCF), and osteopontin on the osteoblast surface by secretion of enzymes, such as matrix metalloproteinase 9 (MMP-9) and cathepsin K [25], suggesting that osteoclasts modulate the endosteal osteoblastic niche. It has also been reported that osteoclasts play a major role in HSC niche formation. Oc/oc mice, in which osteoclasts are inactive due to the loss of normal proton production, leading to severe osteopetrosis, show a dramatic reduction in HSCs and impaired osteoblastic commitment, without deficits in perivascular mesenchymal cells [26]. This result indicates that active osteoclasts are indispensable for endosteal $\mathrm{HSC} /$ HPC niche formation. Another group reported that osteopetrotic mice models, op/op, c-Fos-deficient, and receptor activator of nuclear factor kappa B ligand-deficient mice, in which osteoclasts are deficient and BM cavity is absent, have normal capacity for HSC/HPC mobilization induced by granulocyte colony-stimulating factor (G-CSF) [27]. Therefore, the role of osteoclasts in hematopoietic system remains a matter of debate.

\section{Biology of osteocytes, the third player in bone metabolism}

Osteocytes, terminally differentiated osteolineage cells, are embedded in mineralized bone. Osteocytes arise from MPCs through multiple steps of osteoblast maturation, and are the most abundant osteolineage cells, constituting more than $90 \%$ of all cells in bone [11]. Although these cells were once considered to be inert bystanders surrounded by hard mineralized bone matrix, advances in technology, such as transgenic mouse models, cell lines, and imaging techniques, have enabled the acquisition of new knowledge about function of osteocytes over the past decade. The best known function of osteocytes is mechanosensation. Recently, the osteocyte in vivo depletion model has revealed that osteocytes sense load stress on bone and orchestrate bone remodeling by controlling both osteoblasts and osteoclasts [28]. Osteocytes also play an important role in the metabolism of minerals such as phosphate and calcium, not only locally, but also whole body regulation through the production of sclerostin, dentin matrix protein-1 (DMP-1), phosphate-regulating endopeptidase homolog X-linked (Phex), matrix extracellular phosphoglycoprotein (MEPE), and fibroblast growth factor 23 (FGF23) [11]. Osteocytes extend long dendritic processes that connect with osteoblasts and bone-lining cells on the endosteal surface and neighboring osteocytes [29], and communicate intercellularly through gap junctions, consisting of mainly connexin-43 [30], or by paracrine signaling using small molecules such as prostaglandins, nitric oxide, and adenosine triphosphate through the extracellular fluid in the canaliculi.

\section{Osteocyte and hematopoiesis}

It has been reported that microgravity, which causes loss of bone mass, leads to immune system dysfunction [31, 32]. These changes in hematopoietic cells under microgravity conditions are widely thought to be cell intrinsic. A recent study, however, showed that microgravity disrupts the osteocyte network accompanied with a reduction in B cells in the BM, and that the depletion of osteocytes leads to a decrease in B and T lymphocytes in the blood, due to the impaired development of stromal cells that support B cells in the BM and T cells in the thymus, respectively [14]. Moreover, recent studies have revealed that osteocytes embedded in the bone matrix have critical roles in regulating hematopoietic cells. Fulzele et al. found that osteocytes participate in the regulation of myelopoiesis through the secretion of hematopoietic cytokines, such as G-CSF. They showed that the deletion of Gs $\alpha$ specifically in osteocytes greatly enhances G-CSF production, leading to the expansion of myeloid-committed cells, but not HSCs, in the BM [12].

\section{Role of osteocytes for the mobilization of HSCs/HPCs}

In the clinic, we use the cytokine G-CSF to mobilize HSCs/ HPCs from BM to peripheral circulation. Although the mechanism of how G-CSF mobilizes these immature cells has been investigated intensely, it is still not fully understood. Osteoblasts at the endosteal surface play an essential role in this process [33-35]. Mobilization is finely tuned by complex signals from multiple cell types, such as the sympathetic nervous system (SNS) [34, 36] and BM resident macrophages [37-39]. G-CSF increases sympathetic tone and eliminates endosteal macrophages, which also causes alteration of the HSC/HPC niches. In a recent study, our group assessed osteocyte function in regulating $\mathrm{HSC} /$ HPC mobilization induced by G-CSF [13]. Analysis of gene expression profile of osteoblasts and osteocytes in mice during G-CSF treatment revealed that osteocytes are suppressed by G-CSF treatment in the same manner as osteoblasts. The functional changes in osteocytes appear earlier than those in osteoblasts. Morphological changes in osteocytes following G-CSF treatment, which can be appreciated by using phalloidin staining, are also remarkable. Osteocytes protrude processes enriched in actin 
Fig. 2 Suppression of osteocytes by the treatment of G-CSF. a Phalloidin staining of mouse femoral bone. b G-CSF treatment disrupted the dendritic processes extending from osteocytes to endosteal osteoblasts (a)

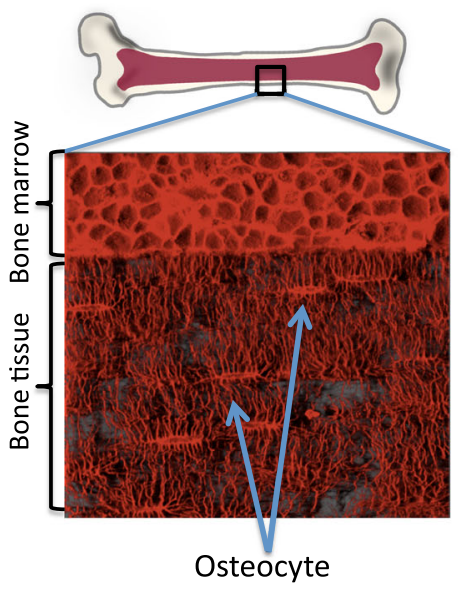

(b)

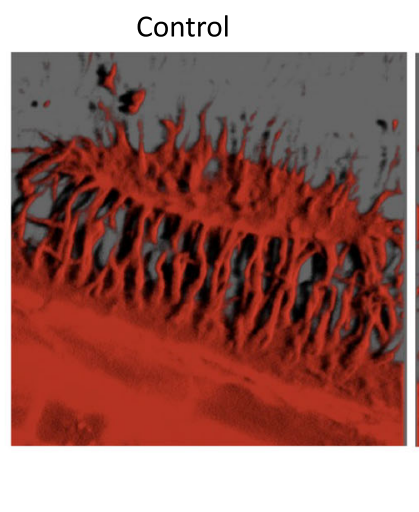

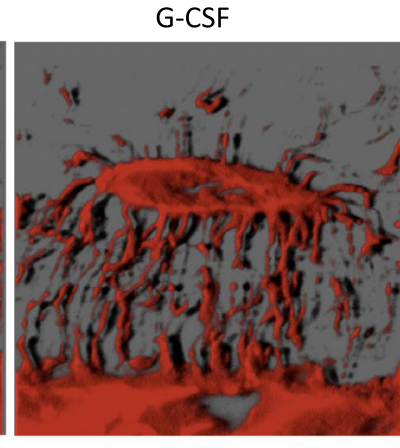

filaments and connect to one another and other cells inside the BM. We found that such osteocyte processes were dramatically thinned in G-CSF-treated animals (see Fig. 2). These results indicate that G-CSF treatment induces transcriptional and morphological changes in osteocytes. Since it has been shown that SNS signals triggered by G-CSF caused osteoblast suppression [34], we hypothesized that SNS may also involved in the mechanism by which G-CSF suppresses osteocyte activity. Surgical denervation of the $\mathrm{BM}$ abolished the gene suppressive effect on osteocytes by G-CSF. This suggests that the function of osteocytes is regulated, at least in part, through the SNS. Together with the increased sympathetic tone following G-CSF treatment, the results raised the possibility that the functional changes in osteocytes could play important roles for HSC/HPC mobilization by G-CSF.

To further explore this issue, we utilized transgenic ( $\mathrm{Tg}$ ) mice, in which osteocytes are specifically ablated through the targeted expression of a diphtheria toxin (DT) receptor (DTR) under a DMP-1 promoter [28]. In this mouse model, we were able to generate osteocyte-less (OL) mice by treatment with DT. In OL mice, G-CSF-induced HSC/HPC mobilization was significantly impaired, while the number of these cells in the BM was unchanged. The number of HSCs/HPCs in the spleen at steady state also decreased. These findings indicate that osteocytes participate in HSC/ HPC egress from the BM to peripheral circulation in steady state and G-CSF-induced mobilization. Since osteocytes functionally communicate with their ancestor, osteoblasts, at the endosteal surface through their dendritic processes [40], we investigated whether the mobilization defect in OL mice was due to impairment of osteoblastic niche function. The depletion of osteocytes led to morphological changes and dysfunctions of osteoblasts. Recently, the role of the BM resident macrophages, so-called osteomacs, located in the endosteum and functioning as an integral player in endosteal niche support, has been reported [39, 41]. The depletion of osteomacs in vivo leads to the disappearance of osteoblasts and the mobilization of HSCs/ HPCs, which suggests that they are indispensable for the endosteal niche [39]. Intriguingly, osteocytes extend dendritic processes into the marrow space near the endosteal surface [42], and osteocyte depletion causes disappearance of osteomacs from the endosteum. These findings raise the possibility that osteocytes are associated with osteomacs as one of the multiple regulatory pathways to control osteoblastic function. In summary, these results indicate that osteocytes control endosteal niche from inside of bone (see Fig. 3). In steady state, the integrity of the endosteal niche is maintained by support signals from both osteomacs and osteocytes. G-CSF administration intensifies the sympathetic tone, which leads to direct suppression of osteoblasts. G-CSF also collapses osteomacs that send support signals to endosteal niche cells. In addition to these pathways, the endosteal niche loses another support signals from osteocytes that are suppressed by SNS signals induced by G-CSF. Eventually, G-CSF mobilizes HSCs/ HPCs by inducing trifold negative effects on the endosteal niche, leading to its collapse. The mechanism how osteocyte regulates osteoblast during G-CSF-induced mobilization is still unclear. Given the fact that conditional deletion of connexin-43 led to dysfunction of osteoblasts and mobilization defect by G-CSF [43], it is conceivable that contact communication through gap junction between osteocytes and endosteal niche cells might be involved in the mechanism of niche regulation.

\section{Osteolineage cells and hematopoietic malignant diseases}

Osteolineage cells play roles not only in normal hematopoiesis but also in hematologic neoplasms. Human acute 


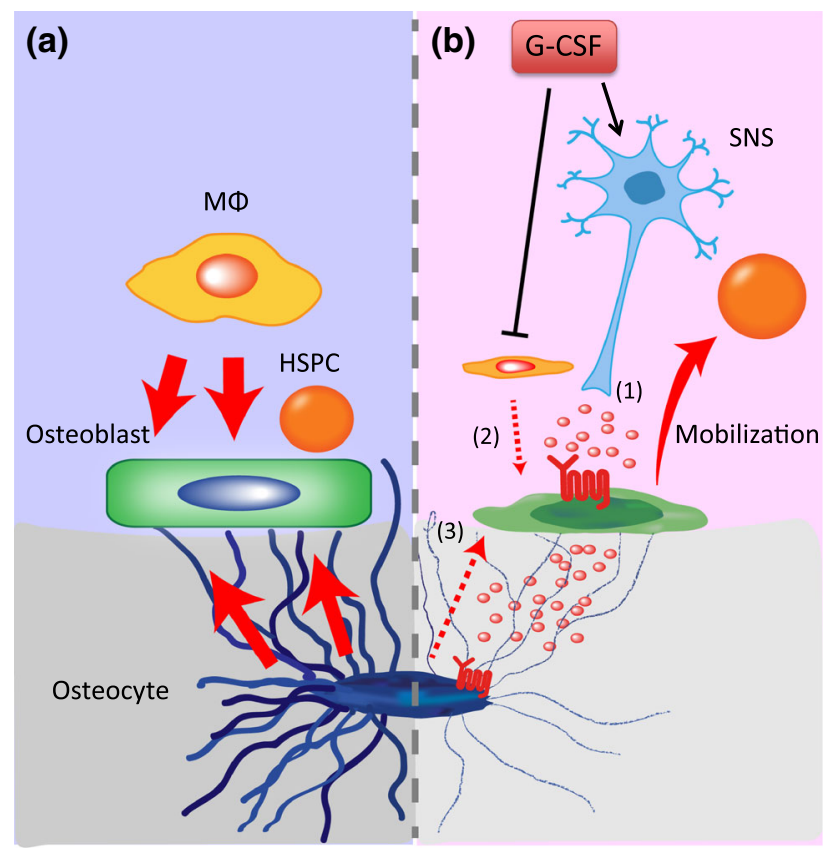

Fig. 3 Osteocytes regulate endosteal niche during G-CSF-induced HSC/HPC mobilization. a In steady state, osteoblasts maintain the niche function by taking supporting signals from osteomacs (MФ) and osteocytes (red solid arrows). b In G-CSF-induced mobilization, osteoblasts are suppressed through three different pathways: 1 direct suppression by SNS-mediated $\beta_{2}$-adrenergic signaling, 2 loss of supporting signals from osteomacs, and 3 loss of supporting signals from osteocytes

myelogenous leukemia (AML) cells engrafted in immunodeficient mice resided in the endosteal area, and the osteoblastic niche promotes the quiescence of AML cells, resulting in their survival after the chemotherapy [44, 45]. It has been reported that leukemic cells remodel the endosteal niche by activating the production of abnormal osteoblastic lineage cells from MPCs, which alters the microenvironment such that it is favorable for leukemic cells and adverse for normal hematopoiesis [46]. An emerging body of evidence suggests that osteolineage cells are involved in the pathogenesis of hematopoietic neoplasms, which had been considered to be hematopoietic cell-intrinsic diseases. The deletion of Dicer1, RNaseIII endonuclease essential for microRNA biogenesis and RNA processing, in Osx positive osteo-progenitor cells in mice caused myelodysplasia, eventually leading to the acute leukemia $[47,48]$. Most recently, constitutive activation of $\beta$-catenin in osteoblasts has been reported to cause AML through the activation of Notch signaling in HSCs/HPCs [49]. These studies provide the evidence that single genetic modification in osteolineage cells can induce hematopoietic malignancy, and further suggests the importance of endosteal microenvironment in the pathogenesis of hematopoietic disorders.

\section{Concluding remarks}

The osteoblastic microenvironment has been studied not only in steady state, but also under "stress" conditions, such as bone marrow transplantation, mobilization, and hematologic disorders. The important roles of each stage of osteolineage cells in regulation of hematopoietic system are becoming increasingly clear. Considering the fact that the entire osteolineage cells from the osteoblast progenitors to the most differentiated osteocytes participate in the regulation of hematopoiesis, the skeletal system may be an important therapeutic target with clinical relevance in hematology field.

Conflict of interest The authors declare that they have no conflict of interest.

\section{References}

1. Omatsu Y, Sugiyama T, Kohara H, et al. The essential functions of adipo-osteogenic progenitors as the hematopoietic stem and progenitor cell niche. Immunity. 2010;33(3):387-99.

2. Mendez-Ferrer S, Michurina TV, Ferraro F, et al. Mesenchymal and haematopoietic stem cells form a unique bone marrow niche. Nature. 2010;466(7308):829-34.

3. Greenbaum A, Hsu YM, Day RB, et al. CXCL12 in early mesenchymal progenitors is required for haematopoietic stem-cell maintenance. Nature. 2013;495(7440):227-30.

4. Ding L, Morrison SJ. Haematopoietic stem cells and early lymphoid progenitors occupy distinct bone marrow niches. Nature. 2013;495(7440):231-5.

5. Kunisaki Y, Bruns I, Scheiermann C, et al. Arteriolar niches maintain haematopoietic stem cell quiescence. Nature. 2013;502(7473):637-43.

6. Komori T, Yagi H, Nomura S, et al. Targeted disruption of Cbfa1 results in a complete lack of bone formation owing to maturational arrest of osteoblasts. Cell. 1997;89(5):755-64.

7. Nakashima K, Zhou X, Kunkel G, et al. The novel zinc fingercontaining transcription factor osterix is required for osteoblast differentiation and bone formation. Cell. 2002;108(1):17-29.

8. Nakashima K, de Crombrugghe B. Transcriptional mechanisms in osteoblast differentiation and bone formation. Trends Genet. 2003;19(8):458-66.

9. Manolagas SC. Birth and death of bone cells: basic regulatory mechanisms and implications for the pathogenesis and treatment of osteoporosis. Endocr Rev. 2000;21(2):115-37.

10. Jilka RL, Weinstein RS, Bellido T, et al. Osteoblast programmed cell death (apoptosis): modulation by growth factors and cytokines. J Bone Miner Res. 1998;13(5):793-802.

11. Bonewald LF. The amazing osteocyte. J Bone Miner Res. 2011;26(2):229-38.

12. Fulzele K, Krause DS, Panaroni C, et al. Myelopoiesis is regulated by osteocytes through Gsalpha-dependent signaling. Blood. 2013;121(6):930-9.

13. Asada N, Katayama Y, Sato M, et al. Matrix-embedded osteocytes regulate mobilization of hematopoietic stem/progenitor cells. Cell Stem Cell. 2013;12(6):737-47.

14. Sato M, Asada N, Kawano Y, et al. Osteocytes regulate primary lymphoid organs and fat metabolism. Cell Metab. 2013;18(5): 749-58. 
15. Taichman RS, Emerson SG. Human osteoblasts support hematopoiesis through the production of granulocyte colony-stimulating factor. J Exp Med. 1994;179(5):1677-82.

16. Calvi LM, Adams GB, Weibrecht KW, et al. Osteoblastic cells regulate the haematopoietic stem cell niche. Nature. 2003;425(6960):841-6.

17. Zhang $\mathrm{J}$, Niu $\mathrm{C}$, Ye L, et al. Identification of the haematopoietic stem cell niche and control of the niche size. Nature. 2003;425(6960):836-41.

18. Lymperi S, Horwood N, Marley S, et al. Strontium can increase some osteoblasts without increasing hematopoietic stem cells. Blood. 2008;111(3):1173-81.

19. Nakamura $\mathrm{Y}$, Arai F, Iwasaki $\mathrm{H}$, et al. Isolation and characterization of endosteal niche cell populations that regulate hematopoietic stem cells. Blood. 2010;116(9):1422-32.

20. Arai F, Hirao A, Ohmura M, et al. Tie2/angiopoietin-1 signaling regulates hematopoietic stem cell quiescence in the bone marrow niche. Cell. 2004;118(2):149-61.

21. Yoshihara H, Arai F, Hosokawa K, et al. Thrombopoietin/MPL signaling regulates hematopoietic stem cell quiescence and interaction with the osteoblastic niche. Cell Stem Cell. 2007;1(6):685-97.

22. Qian H, Buza-Vidas N, Hyland CD, et al. Critical role of thrombopoietin in maintaining adult quiescent hematopoietic stem cells. Cell Stem Cell. 2007;1(6):671-84.

23. Sugimura R, He XC, Venkatraman A, et al. Noncanonical Wnt signaling maintains hematopoietic stem cells in the niche. Cell. 2012;150(2):351-65.

24. Adams GB, Chabner KT, Alley IR, et al. Stem cell engraftment at the endosteal niche is specified by the calcium-sensing receptor. Nature. 2006;439(7076):599-603.

25. Kollet O, Dar A, Shivtiel S, et al. Osteoclasts degrade endosteal components and promote mobilization of hematopoietic progenitor cells. Nat Med. 2006;12(6):657-64.

26. Mansour A, Abou-Ezzi G, Sitnicka E, et al. Osteoclasts promote the formation of hematopoietic stem cell niches in the bone marrow. J Exp Med. 2012;209(3):537-49.

27. Miyamoto K, Yoshida S, Kawasumi M, et al. Osteoclasts are dispensable for hematopoietic stem cell maintenance and mobilization. J Exp Med. 2011;208(11):2175-81.

28. Tatsumi S, Ishii K, Amizuka N, et al. Targeted ablation of osteocytes induces osteoporosis with defective mechanotransduction. Cell Metab. 2007;5(6):464-75.

29. Marotti G, Ferretti M, Muglia MA, et al. A quantitative evaluation of osteoblast-osteocyte relationships on growing endosteal surface of rabbit tibiae. Bone. 1992;13(5):363-8.

30. Yellowley CE, Li Z, Zhou Z, et al. Functional gap junctions between osteocytic and osteoblastic cells. J Bone Miner Res. 2000;15(2):209-17.

31. Crucian B, Sams C. Immune system dysregulation during spaceflight: clinical risk for exploration-class missions. J Leukoc Biol. 2009;86(5):1017-8.

32. Gueguinou N, Huin-Schohn C, Bascove M, et al. Could spaceflight-associated immune system weakening preclude the expansion of human presence beyond Earth's orbit? J Leukoc Biol. 2009;86(5):1027-38
33. Christopher MJ, Link DC. Granulocyte colony-stimulating factor induces osteoblast apoptosis and inhibits osteoblast differentiation. J Bone Miner Res. 2008;23(11):1765-74.

34. Katayama Y, Battista M, Kao WM, et al. Signals from the sympathetic nervous system regulate hematopoietic stem cell egress from bone marrow. Cell. 2006;124(2):407-21.

35. Kawamori Y, Katayama Y, Asada N, et al. Role for vitamin D receptor in the neuronal control of the hematopoietic stem cell niche. Blood. 2010;116(25):5528-35.

36. Mendez-Ferrer S, Lucas D, Battista M, Frenette PS. Haematopoietic stem cell release is regulated by circadian oscillations. Nature. 2008;452(7186):442-7.

37. Chow A, Lucas D, Hidalgo A, et al. Bone marrow CD169+ macrophages promote the retention of hematopoietic stem and progenitor cells in the mesenchymal stem cell niche. J Exp Med. 2011;208(2):261-71.

38. Christopher MJ, Rao M, Liu F, et al. Expression of the G-CSF receptor in monocytic cells is sufficient to mediate hematopoietic progenitor mobilization by G-CSF in mice. J Exp Med. 2011;208(2):251-60.

39. Winkler IG, Sims NA, Pettit AR, et al. Bone marrow macrophages maintain hematopoietic stem cell (HSC) niches and their depletion mobilizes HSCs. Blood. 2010;116(23):4815-28.

40. Taylor AF, Saunders MM, Shingle DL, et al. Mechanically stimulated osteocytes regulate osteoblastic activity via gap junctions. Am J Physiol Cell Physiol. 2007;292(1):C545-52.

41. Chang MK, Raggatt LJ, Alexander KA, et al. Osteal tissue macrophages are intercalated throughout human and mouse bone lining tissues and regulate osteoblast function in vitro and in vivo. J Immunol. 2008;181(2):1232-44.

42. Kamioka H, Honjo T, Takano-Yamamoto T. A three-dimensional distribution of osteocyte processes revealed by the combination of confocal laser scanning microscopy and differential interference contrast microscopy. Bone. 2001;28(2):145-9.

43. Gonzalez-Nieto D, Li L, Kohler A, et al. Connexin-43 in the osteogenic BM niche regulates its cellular composition and the bidirectional traffic of hematopoietic stem cells and progenitors. Blood. 2012;119(22):5144-54.

44. Ishikawa F, Yoshida S, Saito Y, et al. Chemotherapy-resistant human AML stem cells home to and engraft within the bonemarrow endosteal region. Nat Biotechnol. 2007;25(11):1315-21.

45. Ishikawa F. Modeling normal and malignant human hematopoiesis in vivo through newborn NSG xenotransplantation. Int $\mathrm{J}$ Hematol. 2013;98(6):634-40.

46. Schepers K, Pietras EM, Reynaud D, et al. Myeloproliferative neoplasia remodels the endosteal bone marrow niche into a selfreinforcing leukemic niche. Cell Stem Cell. 2013;13(3):285-99.

47. Raaijmakers MH, Mukherjee S, Guo S, et al. Bone progenitor dysfunction induces myelodysplasia and secondary leukaemia. Nature. 2010;464(7290):852-7.

48. Raaijmakers MH. Myelodysplastic syndromes: revisiting the role of the bone marrow microenvironment in disease pathogenesis. Int J Hematol. 2012;95(1):17-25.

49. Kode A, Manavalan JS, Mosialou I, et al. Leukaemogenesis induced by an activating beta-catenin mutation in osteoblasts. Nature. 2014;506(7487):240-4. 